\title{
ESTIMATING WORKLOAD DEMANDS OF TURNING LEFT AT INTERSECTIONS OF VARYING COMPLEXITY
}

\author{
Arne Stinchcombe ${ }^{1}$ and Sylvain Gagnon ${ }^{1,2}$ \\ ${ }^{1}$ School of Psychology, University of Ottawa \\ ${ }^{2}$ CanDRIVE, OHRI \\ Ottawa, Ontario, Canada \\ Email:sgagnon@uottawa.ca
}

\begin{abstract}
Summary: The challenge posed by left-turns has been well-documented in literature. Left-turns are thought to be complex roadway sites resulting in a significant proportion of motor-vehicle collisions. The purpose of the present study was to determine whether subjective and objective workload is affected by left-turns of varying complexity (i.e., information processing and maneuvering) in a sample of young inexperienced drivers. A secondary goal was to determine the effect of administering a secondary task on subjective workload. To this end, 60 inexperienced drivers completed four simulated driving scenarios of varying visual and maneuvering complexity. Half of participants completed an objective measure of workload (i.e., a secondary task) while all participants completed a subjective measure of workload upon completion of each scenario. The results demonstrated the effect of complexity on subjective and objective workload. Specifically, information processing complexity was found to significantly affect both subjective and objective measures of participants' workload while the influence of maneuvering complexity was detected through subjective load only.
\end{abstract}

\section{INTRODUCTION}

It is widely acknowledged that intersections are dangerous roadway sites and account for a significant proportion of motor-vehicle collisions. In fact, crossing path situations, such as leftturns, account for $26 \%$ of all crashes among lightweight vehicles in the United States (Najm, Sen, Smith, Campbell, 2003).

A common explanation for the increased crash rate observed at intersections is that such situations are highly cognitively complex, resulting in greater cognitive workload. The term workload is currently used to describe the set of task demands or the overall level of cognitive resources that are required when completing a task (Gartner \& Murphy, 1979). Specifically, as a result of a greater number of visual stimuli and maneuvering required at a left-hand turn, attentional resources may become overcommitted; thus, the chance of making a driving error (i.e., crash) is more probable.

Several assessments of workload have been proposed and fall largely under subjective estimates (i.e., self-report) and objective measures (i.e., performance-based) (Gawron, 2000). Subjective estimates of workload involve self-report measures subsequent to completing a particular task (e.g., Eggemeier \& Wilson, 1991). On the other hand, objective measures of workload are the most frequently used category and represent the operator's capability to perform the driving task at an acceptable level. Objective estimates are often assessed through primary tasks such as 
lateral and longitudinal control or a secondary task where an extraneous task is used to assess workload (e.g., detection of an unrelated visual stimulus).

The purpose of the present study is to determine whether subjective and objective workload is affected by traffic situations involving left-turns of varying complexity. Participants were assessed in a driver simulator as it provides a safe environment in which to collect data pertaining to workload estimates resulting from changes in maneuvering and information processing complexity (Bella, 2008). It is hypothesized that should difficulties with left-turns arise due to their complexity, differences in workload will follow suit. Specifically individuals should report higher levels of subjective cognitive workload as well as demonstrate slower performance on a secondary task when the complexity (i.e., information processing and maneuvering) of the left turn situation is increased. A secondary objective is to determine the interference generated by a secondary task on driving performance by comparing subjective workload responses of individuals who complete a secondary task to those who do not.

\section{METHOD}

\section{Participants}

A convenience sample of sixty young adults (28 males, 32 females) enrolled in an Introductory Psychology course at the University of Ottawa participated in the present study. Inclusion criteria were a) possession of a valid driver's license and b) less than 25 years of age c) less than 5 years of driving experience. The mean participant age was 18.61 years. All 60 participants completed the testing for this particular study. Participants reported being in good mental and physical health with no neurological, psychiatric or substance abuse problems.

\section{Materials}

\section{Apparatus}

A STISIM driving simulator (Build 3.00.04) produced by Systems Technology Inc. was used to examine the behavioural reactions of participants in simulations. The STISIM driving simulator displays a virtual roadway environment on three wide screens by means of three NEC projectors, giving the driver a field of view of 80 degrees. The simulator is a tool for generating laboratory tasks relevant to the psychomotor and cognitive demands of real-world driving. The simulator allows for the design of urban and suburban roadway environments including interactive vehicles on all lanes, buildings, traffic control devices, and pedestrians through advanced vehicle dynamics and image generation. The virtual environment is supplemented with realistic audio effects providing acceleration cues. Also, the driving simulator includes an optional dual task (DT), which consists of visual stimuli appearing in the periphery. The participant is required to detect the stimulus on either the left or right sides of their field of view as soon as it is perceived by signalling left (downward motion) or right (upward motion) with the signaller located to the left of the steering wheel. The software was run on a Windows XP operating system and Dell computers with a processing speed of $2394 \mathrm{mhz}$ (four systems required). 


\section{Scenarios}

Participants completed four versions of the same basic scenario that differed in complexity. Specifically, the basic scenario consisted of a $1 \mathrm{~km}$ route with an intersection located at 500 meters where participants were instructed to turn left. The complexity of the scenario was manipulated according to information processing and vehicle handling which was adapted from Fastenmeier's (1995) classification scheme. This taxonomy produced four derivatives of the same scenario.

The information processing manipulation consisted of two levels (i.e., high vs. low). The high level was constructed so as to mimic an urban city-centre with traffic, parked cars, pedestrians, and tall office buildings; the intersection was controlled by a traffic light. On-coming traffic was presented at the intersection. In the low level, the scenario was constructed so as to have as little visual information as possible (Fig. 1). In particular, there were no buildings, trees, parked cars or pedestrians; the intersection consisted of a stop sign. The sole traffic was an oncoming vehicle located at the intersection. The maneuvering manipulation also consisted of two levels (i.e., high vs. low). The high level consisted of a series of lane changes. Specifically, scenarios that were high in maneuvering required participants to lane change four times (twice before the intersection and twice after).
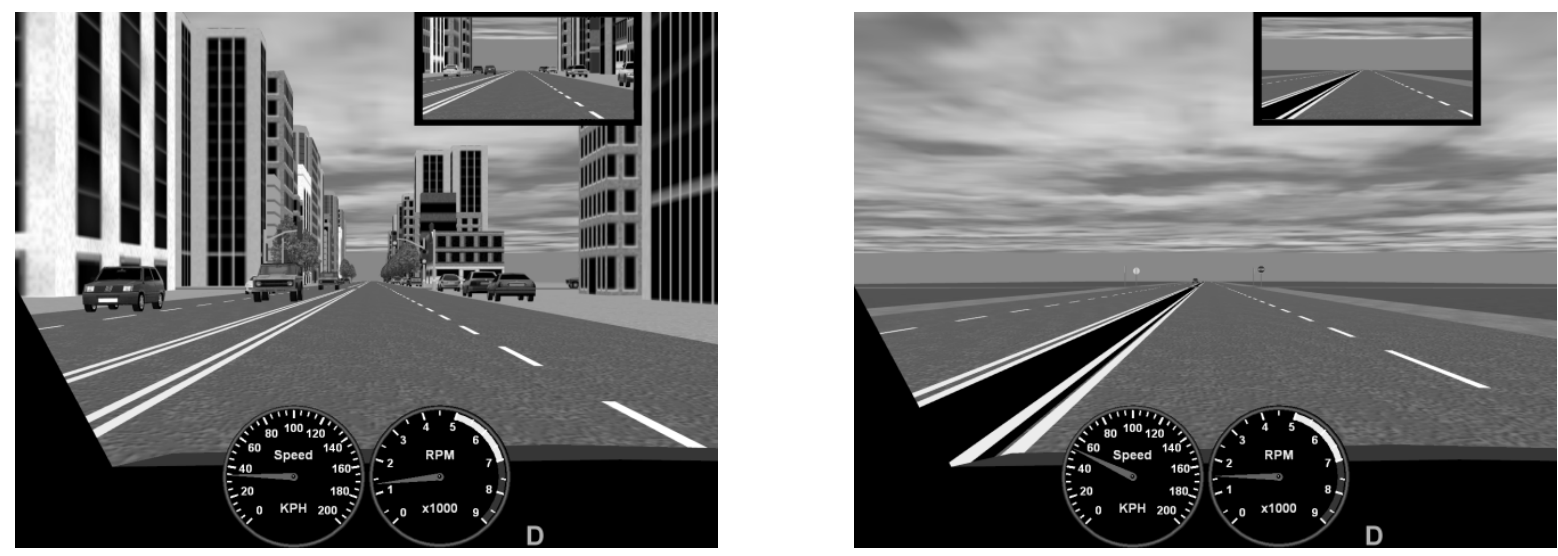

Fig 1. High information processing scenario (left) and low information processing scenario (right)

The resulting manipulations were combined and resulted in four distinct scenarios: 1) High demands on information processing and high demands on vehicle handling 2) High demands on information processing and low demands on vehicle handling 3) Low demands on information processing and high demands on vehicle handling 4) Low demands on information processing and low demands on vehicle handling.

\section{Objective measure of workload}

Half of the participants were placed in a dual task condition. Specifically, for this group each of the four scenarios contained a DT presented at 505 meters (i.e., in the middle of the intersection) and either 250 meters (i.e., prior to the intersection) or 750 meters (i.e., subsequent to the intersection). The participants were given 5 seconds to react to the visual DT stimulus after which it disappeared. 


\section{Subjective measure of workload}

The NASA-TLX was employed as a measure of subjective cognitive load. The NASA-TLX is a standard subjective workload measure and it is regarded as a sensitive and reliable measure to assess subjective workload (Hart \& Staveland, 1988). This instrument is comprised of six subscales (mental demand; temporal demand; physical demand; effort; frustration; performance) with possible scores ranging from one to 21. A weighting procedure is included in the complete NASA-TLX to generate a composite score but for the purpose of this study, scores on each of the six individual subscales were separately analyzed, a procedure that has shown to generate similar results (Byers, Bittner, \& Hill, 1989).

\section{Procedure}

Prior to arriving in the laboratory participants were randomly assigned to a DT condition or a non-DT condition. Participants arrived at the lab and completed an informed consent document. They subsequently completed a 10-minute orientation session of increasing complexity, allowing them to familiarize themselves with maneuvering in the simulator. Thereafter, participants completed a series of 20 independent scenarios of varying complexity including the four we report here. Participants were instructed to maintain a speed of $50 \mathrm{~km} /$ hour throughout but were also told to reduce their speed when they felt it was necessary to do so. The order of presentation was randomized between participants. Following each scenario, participants completed the NASA-TLX questionnaire.

\section{RESULTS}

In order to determine the effect of DT and scenario complexity on subjective cognitive load in response to a left hand turn, six mixed ANOVA were executed for each subscale of the NASATLX (i.e., mental demand, temporal demand, physical demand, effort, frustration, performance). Specifically, $2 \times 2 \times 2$ mixed designs were employed with one between subjects factor which was condition (i.e., presence vs. absence of DT) and two within subjects measures factors which were information processing (i.e., high vs. low), and maneuvering (i.e., high vs. low)). Results indicated significant effects of information processing (IP) and maneuvering (MA), for mental demand [IP: $\mathrm{F}(1,58)=7.48, \mathrm{p}=.008, \mathrm{MA}: \mathrm{F}(1,58)=10.53, \mathrm{p}=.002]$, physical demand [IP: $\mathrm{F}(1$, $58)=4.42, \mathrm{p}=.040, \mathrm{MA}: \mathrm{F}(1,58)=4.70, \mathrm{p}=.034]$, and temporal demand $[\mathrm{IP}: \mathrm{F}(1,58)=5.070$, $\mathrm{p}=.028$, MA: $\mathrm{F}(1,58)=11.89, \mathrm{p}=.001]$. Significant effects of IP were also observed for the frustration subscale, $F(1,58)=8.11, p=.006$ ( see Fig 2). No effect of the presence of the DT was observed and no interactions reached significance. Maneuvering and information processing did not reach significance for the performance and effort subscales.

Subsequently, so as to determine the effect of scenario complexity on DT performance, a within subjects ANOVA was executed, employing the response time to the DT as a dependent variable. Specifically, a 2x2x2 design was employed with information processing (i.e., high vs. low), maneuvering (i.e., high vs. low) and time (i.e., pre/post intersection vs. during intersection). Maneuvering did not yield a significant result $F(1,23)=1.67, p=.209$, suggesting that changing lanes does not significantly impact participants' DT performance. A significant interaction effect was observed between information processing and time indicating that the effect of information 
processing depends on when the DT is presented, $F(1,23)=53.67, p<.001$. To tease apart the interaction, simple main effects were computed by separating DT performance for the two levels of information processing (i.e., high vs. low) by time (i.e., pre/post intersection vs. during intersection). The results indicated a significant effect of information processing complexity during the intersection, $F(1,26)=53.92, p<.001$, but not for pre/post intersection, $F(1,27)=0.82$, $p=.777$. This finding suggests that participants' DT responses were significantly slower during the high information processing scenarios solely during the event; performance did not vary significantly before or after the event when information processing was manipulated.

a)

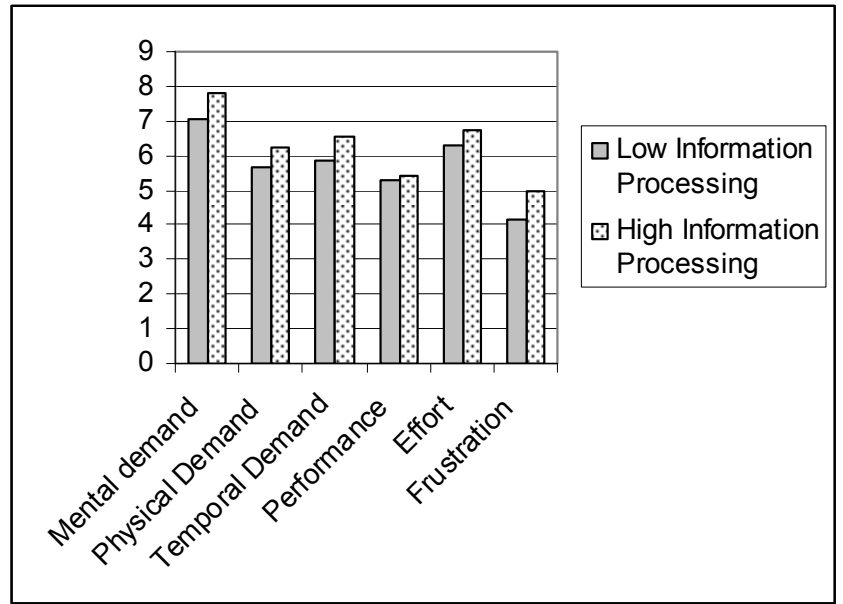

b)

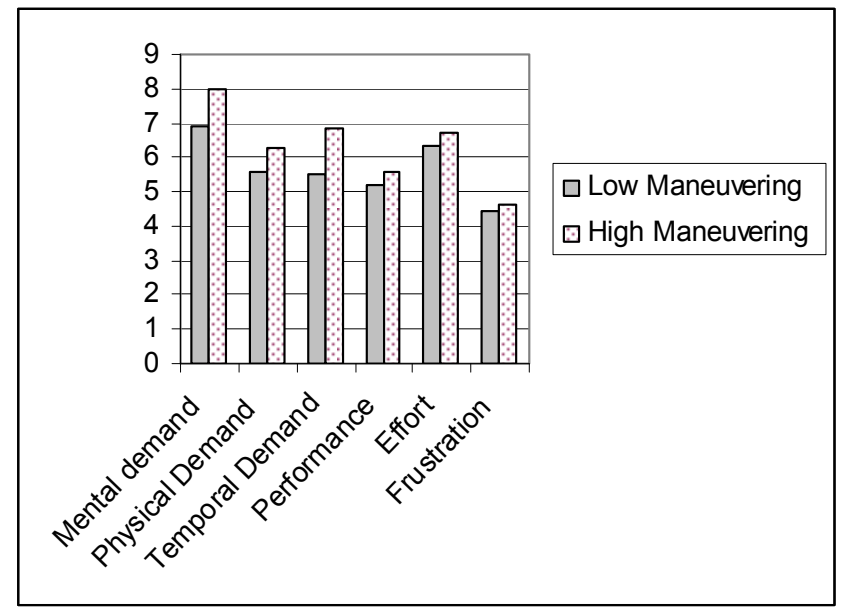

Fig. 2. Means NASA-TLX subscales presented for a) information processing and b) manoeuvring effects.

\section{DISCUSSION}

The present study sought to investigate the role of complexity on objective and subjective workload among inexperienced drivers in response to a scenario in which motor-vehicle collisions often occur. The study yielded several noteworthy findings.

First, consistent with our hypothesis, subjective workload was significantly affected by both information processing and maneuvering manipulations. In particular, individuals rated highly complex scenarios as requiring more overall workload than those with minimal complexity. Secondary task performance indicated significantly slower response times in high information processing scenarios but not in scenarios categorized as high in maneuvering. This finding suggests that both of the complexity manipulations presented here are sufficient to influence participants' subjective appraisals of how difficult the scenario was. However, the maneuvering manipulation was not sufficient to induce deficits on a secondary task. This finding may be due to the maneuvering manipulations occurring prior to as well as subsequent to the intersection and, which suggests that the DT may exclusively capture workload at the left-turn. Following this interpretation, these two measures (i.e., objective and subjective) seem to provide a similar account of workload in these scenarios. 
Third, the presentation of a secondary task had no significant effect on subjective workload. This finding suggests that the secondary task described here did not significantly affect participants' subjective appraisals of workload and indicates its appropriateness for use with this population. Specifically, the presence of a secondary task does not significantly interfere with driving performance over and above the act of driving, suggesting that it may be a pure measure of workload rather than a noteworthy contributor.

The present study was successful in demonstrating the effect of complexity on workload in an inexperienced sample of young drivers when completing left-turns. However, this query unveiled some additional research questions. The present study focused solely on one primary scenario; it would be interesting to examine workload demands of different road-way configurations. Also, to further explore the usefulness of the secondary task, it would be of interest to compare primary task performance (e.g., lateral and longitudinal control) between individuals in the DT and non-DT conditions. Finally, the secondary task proved useful in measuring workload with a group of young drivers; however, it remains unclear whether this finding is replicable across age groups. Specifically, using the secondary task with older adults, with their associated slower processing speed and deficits in visual attention, may prove difficult as it may significantly impact their subjective workload ratings, and even their primary driving performance.

\section{ACKNOWLEDGMENTS}

This research was supported by grants from the Ontario Neurotrauma Foundation and the Natural Sciences and Engineering Research Council of Canada (NSERC) to Sylvain Gagnon. Arne Stinchcombe is supported by a Canadian Institutes of Health Research (CIHR) doctoral scholarship.

\section{REFERENCES}

Bella, F. (2008). Driving simulator for speed research on two-lane rural roads. Accident Analysis and Prevention, 40, 1078-1087

Byers, J. C., Bittner, A. C., \& Hill, S. G. (1989). Traditional and raw task load index correlations: Are paired comparisons necessary? In A. Mital (Ed.), Advances in industrial ergonomics and safety (pp. 481-485). Taylor \& Francis: London.

Eggemeier, F. T., \& Wilson, G. F., (1991). Performance-based and subjective assessment of workload in multi-task environments. In D.L. Damos (Ed.) Multiple-task performance (pp. 207-216). Taylor Francis: London.

Fastenmeier, W. (1995). Die Verkehrssituation als Analyseeinheit im Verkehrssystem. In: Fastenmeier, W. (Hrsg.), Autofahrer und Verkehrssituation (pp. 27-78.). Verlag TÜV Rheinland, Köln.

Gartner, W.B. \& Murphy, M.R. (1979). Concepts of Workload. In B.O Hartman and R.E. McKenzie (Eds.) Survey methods to assess workload.

Gawron, V. J. (2000) Human Performance Measures Handbook . Lawrence Erlbaum Associates, London, UK. 
Hart, S., \& Staveland L. (1988) Development of NASA-TLX (Task Load Index): Results of empirical and theoretical research. In: Hancock P, Meshkati N (eds). Human Mental Workload (139-183). North Holland, Amsterdam.

Najm,W. Sen, B. Smith, D., \& Campbell B. (2003). Analysis of light vehicle crashes and precrash scenarios based on the 2000 General Estimates System. Washington, DC: National Highway Traffic Safety Administration, US Department of Transportation.

Wang, X., \& Abdel-Aty, M. (2008). Analysis of left-turn crash injury severity by conflicting pattern using partial proportional odds models. Accident Analysis \& Prevention. 40, 5, 16741682. 